\title{
Orbital Emphysema
}

National Cancer Institute

\section{Source}

National Cancer Institute. Orbital Emphysema. NCI Thesaurus. Code C127820.

The presence of air or gas in the subcutaneous tissue of the orbit of the eye. 